\title{
Impacto de las unidades de selva manejada tradicionalmente en la conectividad del paisaje de la Sierra de Los Tuxtlas, México
}

Yunin Aguilar Vásquez ${ }^{1}$, Mario Manuel Aliphat Fernández ${ }^{1}$, Laura Caso Barrera ${ }^{1}$, Silvia del Amo Rodríguez ${ }^{2}$, María de Lourdes Sánchez Gómez ${ }^{3} \&$ Daniel Martínez- Carrera ${ }^{1}$

1. Colegio de Postgraduados- Campus Puebla. Carretera Federal México-Puebla km 125.5, Santiago Momoxpan, Municipio de San Pedro Cholula, Puebla, C. P. 72760, México; yuninav@hotmail.com, marioaliphat@yahoo.com, lauracaso2004@yahoo.com, dcarrera@colpos.mx

2. Centro de EcoAlfabetización y Diálogo de Saberes, Universidad Veracruzana- Campus USBI. Avenida de las Culturas Veracruzanas No. 1, Col. Emiliano Zapata, Xalapa, Veracruz, C. P. 91060, México; sdelamo@uv.mx, sdelamoro@gmail.com

3. Universidad Autónoma de Tlaxcala. Av. Universidad No.1, Col. La Loma Xicohténcatl, Tlaxcala, Tlaxcala, C. P. 90070, México; 1ulismex@gmail.com

Recibido 12-XII-2013. Corregido 12-III-2014. Aceptado 19-IV-2014.

\begin{abstract}
Impact of traditionally managed forest units on the landscape connectivity of Sierra de Los Tuxtlas, Mexico. The ever-increasing establishment of landscape mosaics is expressed as a surrounding matrix of agricultural activities, which frames patches or remnants of the original vegetation cover. Conservation actions should be aimed to establish or to increase those interactive systems, which help to maintain the landscape flow through linkages. Spaces occupied by traditional management systems retain and support this function. In this paper, we used Geographic Information Systems to evaluate the importance of traditionally managed forest units ('acahuales'-coffee plantations) and to assess landscape connectivity in the indigenous Popoluca area of Sierra de los Tuxtlas, Mexico. The cartographic material used to establish the types of vegetation and their coverture included the period 1991-2008. At landscape level, four indices were used to assess the general situation of the habitat network, and to identify the patches of high priority. Individually, indices evaluated if patches were important for their area, their potential flow or their connecting function. Results showed that the landscape is functioning as a single system, but having low connectivity. Values improved when traditionally managed forest patches were considered as viable habitat. We detected 367 patches of very high priority, $80 \%$ belonging to forests managed traditionally. Patches were important for their potential flow (size and topological relationships). Only 70 patches were significant for their function as biological corridors between largest forests located at the top of the volcanoes, and are mostly managed forest (75\%). We concluded that the units of traditionally managed forest play a significant role in landscape connectivity maintenance. Rev. Biol. Trop. 62 (3): 1099-1109. Epub 2014 September 01.
\end{abstract}

Key words: tropical forest, 'acahuales', landscape connectivity, Popoluca traditional management, Los Tuxtlas.

Los ecosistemas tropicales experimentan una pérdida masiva de diversidad biológica además de procesos de fragmentación de hábitats, resultado de la introducción de sistemas intensivos y extensivos de producción agrícola. Estos cambios desencadenan transformaciones profundas que disminuyen el tamaño y la conectividad de los hábitats naturales y son la causa de la pérdida de biodiversidad (Santos
\& Tellería, 2006; Kettunen, Terry, Tucker, \& Jones, 2007). La cobertura de la vegetación natural se está convirtiendo en un mosaico de diversos tamaños de vegetación natural, que son relictos de ambientes que han sido remplazados por sistemas menos diversos y de estructuras ecológicas simplificadas. Junto a esta problemática se encuentra el reto de mantener y conservar la biodiversidad en este 
tipo de paisajes (McIntyre \& Hobbs, 1999; Bennett, 2004).

Actualmente, se observa que segmentos múltiples de hábitats que funcionan juntos como un sistema interactivo, son un medio eficaz de conservación; ya que mantienen los flujos naturales del paisaje. Se considera necesaria la conservación a largo plazo por medio de sistemas integrados como redes conformadas por hábitats protegidos y no protegidos, unidos por enlaces de paisaje (Bennett, 2004; Chazdon et al., 2009). Es en este contexto, que encontramos con frecuencia espacios manejados por grupos étnicos como el de los popolucas de la Sierra de los Tuxtlas (SLT), en Veracruz, México. Este grupo indígena tiene formas de producción agrícola y de manejo de recursos naturales disponibles con un bajo nivel de intensidad, que no compromete la función del enlace. Si bien, la importancia y función de los espacios manejados por grupos étnicos empieza a ser reconocida y estudiada, (Anderson, 2003; Toledo, Ortiz, Cortés, Moguel, \& Ordoñez, 2003; Boege, 2008), pero aún dista mucho de alcanzar la trascendencia y reconocimiento que merecen.

Estos espacios de bajo impacto ambiental son manejados por comunidades campesinas e indígenas, con base en su conocimiento y prácticas tradicionales, que reconocen la heterogeneidad ambiental (Altieri, 1991; Berkes, Colding, \& Folke, 2000; Gliessman et al., 2007; Reyes-García, Martí, McDade, Tanner, \& Vadez, 2007). Entre las ventajas que ofrecen los sistemas tradicionales es maximizar la diversidad y el número disponible de opciones, con el fin de garantizar su subsistencia y minimizar los riesgos. Estos procesos culturales se construyen mediante el uso múltiple del espacio, el tiempo, las poblaciones y comunidades de organismos (Gadgil, Berkes, \& Folke, 1993; Toledo et al., 2001; Gliessman, 2002; Altieri, 2004; Pohle \& Gerique, 2006; Boege, 2008). Las regiones indígenas de México y en particular las del trópico húmedo son verdaderas zonas de conservación in situ del patrimonio biológico y cultural de México (Toledo, Ortiz,
\& Medellín- Morales, 1994; Gómez Pompa \& Kaus, 1998; Boege, 2008).

En este contexto, el objetivo de este trabajo fue comprobar por medio de sistemas de información geográfica (SIG’s), si las unidades de selva manejada por las comunidades popolucas (acahuales-cafetales) favorecen la conectividad entre los fragmentos de vegetación natural en el paisaje de la SLT.

\section{MATERIALES Y MÉTODOS}

Área de estudio: El análisis comprendió el área reconocida de ocupación Popoluca (Velázquez, 2001) (18²2'5" N - 9454'48" W), en la Sierra de Santa Marta, Veracruz, México. La zona está dentro de la SLT, la cual fue declarada como Reserva de la Biosfera en 1998 (SEMARNAT, 1998). Forma parte del Eje Volcánico Transversal, sin embargo, se levanta como un macizo volcánico aislado, rico en características ambientales, que se reflejan en una importante diversidad biológica (Arriaga et al., 2000). Esta zona forma parte del área cultural mesoamericana, que representa una gran riqueza cultural y lingüística; además de ser uno de los principales centros de origen de plantas cultivadas y de diversidad biológica (Toledo et al., 2001; Boege, 2008). Los grupos indígenas presentes en la SLT son popolucas y nahuas del sur de Veracruz (Boege, 2008; Lewis, Simons, \& Fenning, 2013). En el año 2000, en el estado de Veracruz, la población popoluca ascendía a 53214 hablantes de esta lengua, y la población nahua a 60470 ; lo que representaba $97.5 \%$ y $3.6 \%$ del total nacional respectivamente (CDI, 2006). Ambos grupos tienen un profundo conocimiento sobre el uso diversificado de los recursos, así como concepciones mágico-religiosas que rigen el aprovechamiento de los mismos (Guevara Sada, Laborde Dovalí, \& Sánchez Ríos, 2000a; Toledo et al., 2001; CONANP, 2006). La zona enfrenta serios problemas ambientales por el crecimiento demográfico, la ganaderización, la extracción ilegal de especies y la deforestación, entre otras amenazas. Esta situación ha provocado una severa fragmentación del paisaje 
que pone en riesgo la diversidad biológica y la continuidad de las prácticas indígenas tradicionales (Paré, 1994; Paré, Agüero, \& Blanco, 1994; González, Dirzo \& Vogt, 1997; Arriaga et al., 2000; Guevara Sada, Sánchez Ríos, \& Landgrave, 2004; CONANP, 2006).

Hábitat viable: Para fines del presente trabajo, se interpretó como hábitat viable toda aquella unidad espacial de un mínimo de $3 \mathrm{ha}$, que contara con la estructura y composición de una vegetación primaria conservada, o secundaria en regeneración con un manejo de baja intensidad; y por tanto, un espacio potencial para la existencia de biodiversidad y el desarrollo de procesos ecológicos en el paisaje. Con base en las descripciones de Castillo-Campos y Laborde (2004), las áreas de acahuales de selva, cafetales con acahual y dosel de selva y áreas de vegetación natural conservada (es decir, selva perennifolia alta y mediana, bosque mesófilo de montaña, bosque de encinar cálido, bosque de pino o manglar) existentes en la zona de estudio, se asumieron como unidades de hábitat viable. Para establecer dichas áreas, se elaboró un mapa de vegetación y uso de suelo, mediante la clasificación supervisada de una imagen de satélite Landsat 7-ETM 2000. Se definieron sólo las categorías suficientes que permitieran identificar exclusivamente los tipos de vegetación antes mencionados. La clasificación se verificó con ortofotos de la zona escala 1:40 000 (INEGI, 2008) y el mapa de vegetación y uso de suelo de Castillo-Campos y Laborde (2004); mismo que se basa en foto aérea de INEGI del año 1991 y recorridos de campo y colectas de ejemplares de los años 1994 y 1998. Considerando que, la tasa de deforestación estimada para los años 20072011 fue de 0.32\% (PSSM, 2011), es posible extrapolar la conectividad evaluada al presente.

Índices de conectividad: Para evaluar la conectividad del hábitat viable del paisaje se calcularon los índices: número de enlaces (NL), número de componentes (NC), índice de conectividad integral (IIC) e índice de probabilidad de conectividad (PC); a través del programa Conefor (Saura \& Torné, 2009). NL indica el total de conexiones directas entre las unidades. NC indica el número de los grupos que existen, asumiendo que en un grupo hay conexiones entre cada uno de los posibles pares de unidades. IIC es un índice de disponibilidad de hábitat, porque combina los atributos con el número de enlaces en la ruta más corta entre cada posible par. PC también es un índice de disponibilidad de hábitat, combina los atributos con el máximo producto de probabilidad de todas las posibles rutas entre cada uno de los pares. Representa la probabilidad de que dos elementos tomados al azar del paisaje estén interconectados, en el marco de un grupo y las conexiones que en él se dan (Pascual-Hortal \& Saura, 2006; Saura \& Pascual-Hortal, 2007; Saura \& Torné, 2009).

Como requisito para los cálculos se definieron ciertas variables. El atributo empleado para caracterizar las unidades fue el área, por considerarse la información descriptiva más sencilla y útil (Arroyo-Rodríguez \& Mandujano, 2006; Saura \& Pascual-Hortal, 2007; McGarigal, 2014). Se usaron como distancias de desplazamiento o capacidad dispersiva, dos (corto), seis (medio) y $12 \mathrm{~km}$ (largo), congruentes con el hábitat de diversa fauna descrita en la zona (Estrada \& Coates-Estrada, 1995; González et al., 1997; González \& Vicario, 1998; Villavicencio, Saura, Santiago, \& Chávez, 2009); y una probabilidad de dispersión de 0.5 (Pascual-Hortal \& Saura, 2007; Neel, 2008). Se corrieron dos rutinas a nivel paisaje, la primera (A) donde se consideraron solo los fragmentos correspondientes a la vegetación natural conservada; y la segunda (B) donde además se tomaron en cuenta los fragmentos de acahuales y cafetales.

Posteriormente, para determinar cuáles son las unidades de hábitat viable más importantes para mantener la conectividad del paisaje, se estimaron los índices IIC y PC a nivel individual (Pascual-Hortal \& Saura, 2006; Saura \& Pascual-Hortal, 2007; Saura \& Torné, 2009). Los resultados se dividieron en cinco categorías (importancia para la conectividad muy alta, alta, media, baja y muy baja). Se consideraron 
como de interés sólo aquellas de muy alta importancia para la conectividad. En ellas se evaluó si favorecen la conectividad del paisaje por el área que tienen (bloque intra), por el flujo potencial que pueden mover debido a su área y a sus relaciones topológicas (bloque flujo), o por su función como conectores y posibles elementos de un corredor de tipo trampolín, dadas sus relaciones topológicas (bloque conector) (Saura \& Rubio, 2010). Finalmente, se consideró que el porcentaje de las unidades de muy alta importancia que actúan como conectores, son de selva manejada.

\section{RESULTADOS}

La zona de estudio comprendió un área de 224 023.217ha. El mapa de vegetación y uso de suelo resultante de la clasificación contó con siete clases generales para la zona, cuatro clases de coberturas naturales y tres antropizadas en distinto grado de manejo (Fig. 1). Los fragmentos menores a 3ha cubrieron 25 475.06ha (11\% de la superficie total), y fueron en su mayoría de selva y selva manejada. Del área restante, la clase que cubrió la mayor extensión fue la zona agropecuaria (119 167.775ha, 53\%), principalmente con fragmentos de amplia superficie, por tanto representó la matriz del paisaje. Le siguió en superficie la selva manejada (24 945.901ha, $11 \%)$, la cual mostró una alta fragmentación (37.5\% de los fragmentos tuvieron menos de 1ha). Las coberturas naturales representaron un bajo porcentaje del paisaje (manglar 457.215 ha- $0.2 \%$, bosques 6557.957 ha- $2.92 \%$ y selvas 12 156.538ha- 5.4\%) y se distribuyeron como grandes relictos en las partes elevadas de los volcanes, y como fragmentos

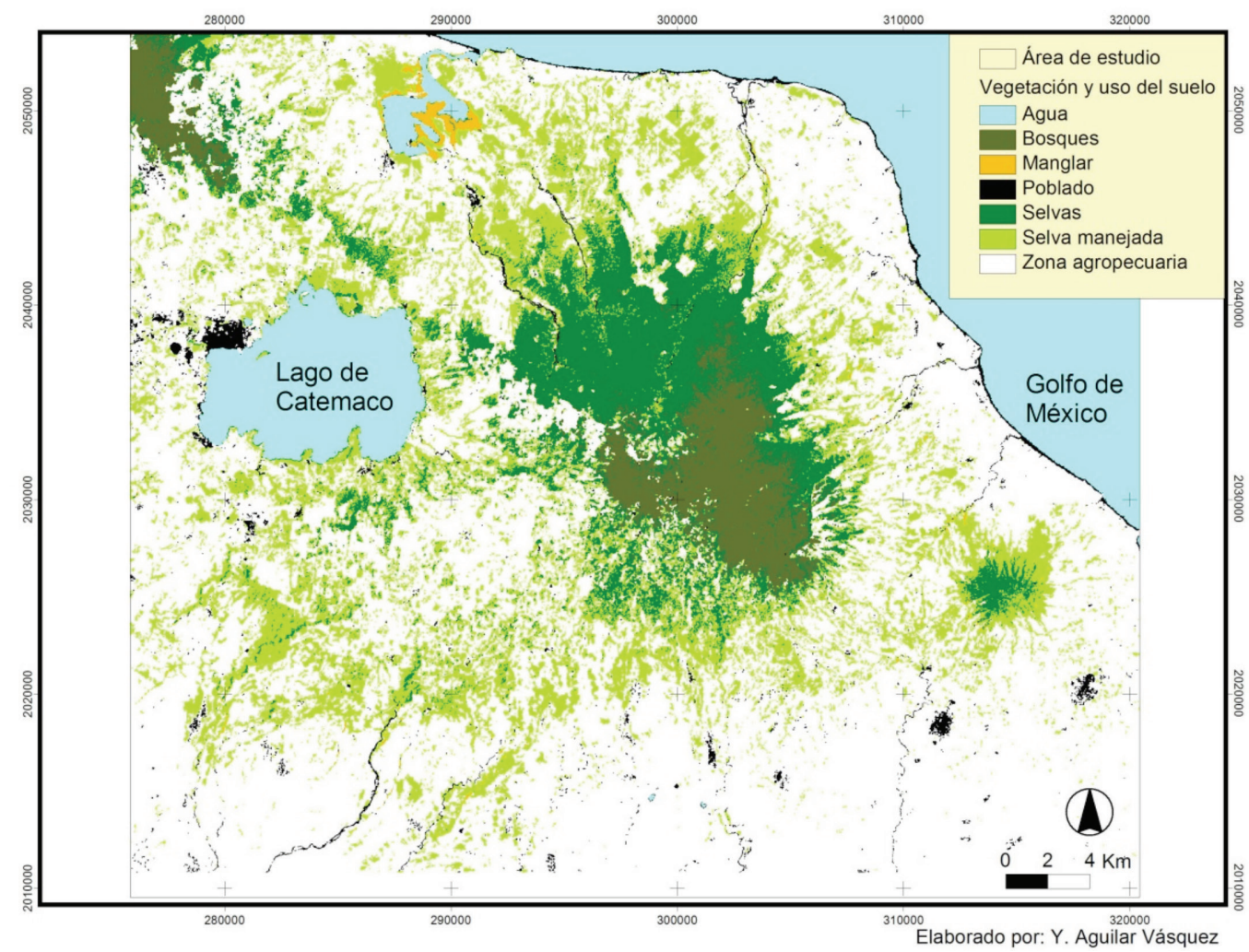

Fig. 1. Vegetación y uso del suelo. Clasificación supervisada de la vegetación usando imagen de satélite LANDSAT 7ETM2000, mapa de vegetación y uso de suelo de Castillo-Campos y Laborde (2004) y ortofotos de INEGI (2008).

Fig. 1. Vegetation and land use. Supervised classification of vegetation using LANDSAT 7ETM-2000 satellite image, Castillo-Campos and Laborde vegetation and land use map (2004) and INEGI orthophotos (2008). 
pequeños dispersos $(33.7 \%$ tuvieron menos de 1 ha) en las partes bajas. Los cuerpos de agua y poblados cubrieron $16.5 \%$ del área.

De acuerdo con esta clasificación, se detectaron 1479 unidades de hábitat viable, cubriendo un área de 44 117.613ha, lo que representó un $20 \%$ del total del paisaje (Fig. 2). De ellas solo un $20 \%$ fue de vegetación natural conservada, el resto fue de selva manejada. La mayoría (97\%) tuvo menos de 100ha de superficie, solo $0.4 \%$ tuvo más de $1000 \mathrm{ha}$; de las cuales solo tres representaron vegetación natural conservada.

En el cuadro 1 se muestran, a modo comparativo, los valores para ambos mapas de los índices calculados. Los valores de conectividad para ambos mapas fueron bajos, considerando que los índices IIC y PC oscilan entre cero y uno, sin embargo, fue posible notar un importante avance cuando se incluyó la selva manejada como hábitat viable y lo mismo sucedió con el número de enlaces. En rangos de dispersión medio y largo, el paisaje se comportó como uno solo, sin embargo, para especies de desplazamiento corto, los relictos de vegetación natural conservada por si solos ya no funcionaron como un paisaje continuo. Por el contrario, funcionaron como si fueran 11 distintos, algunos de varios elementos y otros con solo uno. Esta situación se optimizó al incluir la selva manejada, ya que aunque inicialmente este se expresó como contando con cuatro componentes, en realidad el sistema funcionó como uno, con tres elementos, debido a que originalmente estaban alejados y actuaron en el análisis como unidades aisladas.

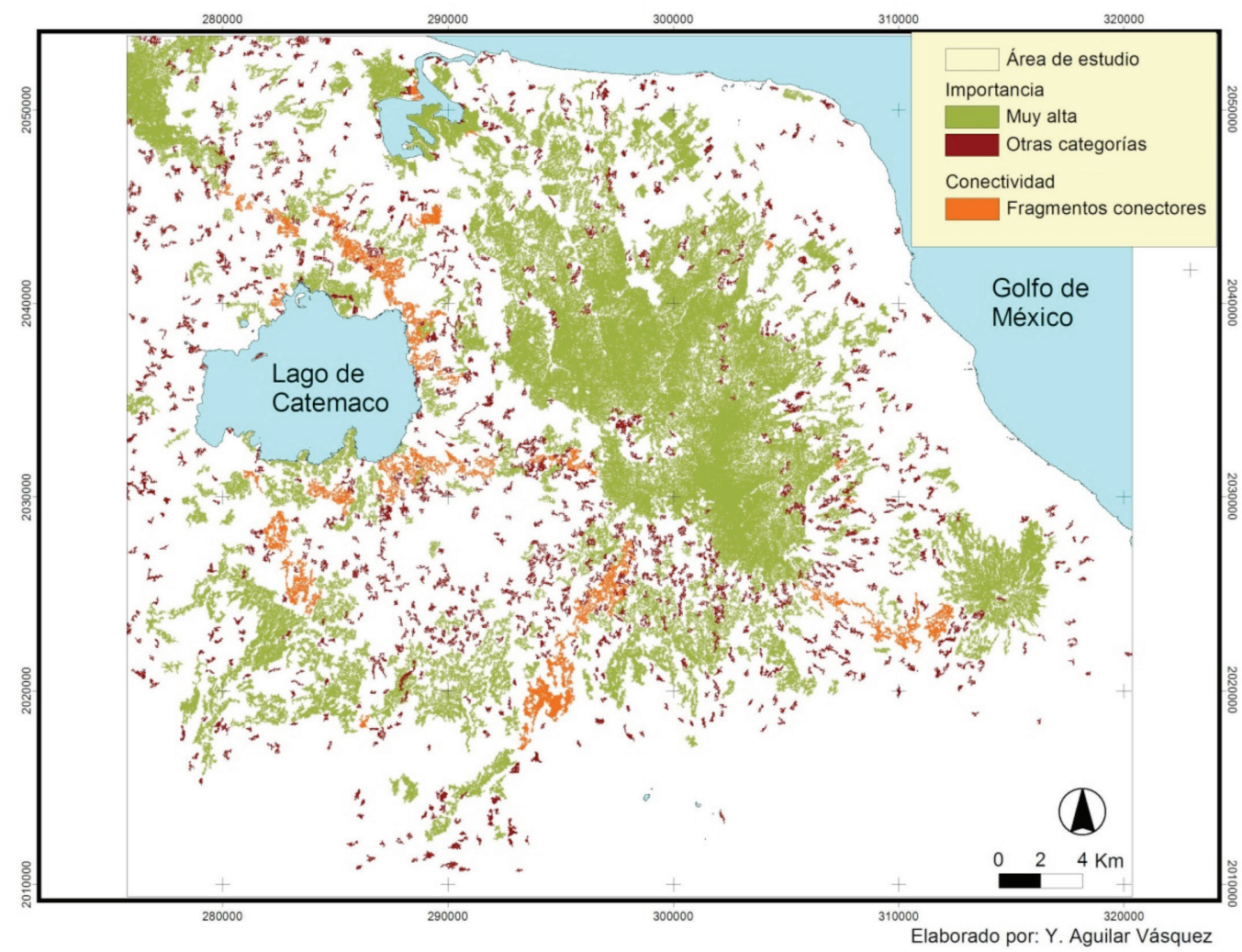

Fig. 2. Hábitat viable, fragmentos de muy alta importancia para la conectividad y fragmentos que cumplen el papel de conectores.

Fig. 2. Viable habitat, fragments of very high importance for connectivity and fragments with a connector role. 
CUADRO 1

Índices de conectividad

TABLE 1

Connectivity indices

\begin{tabular}{|c|c|c|c|}
\hline Capacidad dispersiva & Índices de conectividad & Rutina A & Rutina B \\
\hline \multirow[t]{4}{*}{$12 \mathrm{~km}$} & $\mathrm{NC}$ & 1 & 1 \\
\hline & NL & 20337 & 292733 \\
\hline & IIC & 0.0062145 & 0.0243871 \\
\hline & $\mathrm{PC}$ & 0.0094478 & 0.0540837 \\
\hline \multirow[t]{4}{*}{$6 \mathrm{~km}$} & $\mathrm{NC}$ & 1 & 1 \\
\hline & NL & 8675 & 99794 \\
\hline & IIC & 0.0057067 & 0.0202194 \\
\hline & $\mathrm{PC}$ & 0.0087178 & 0.0525823 \\
\hline \multirow[t]{4}{*}{$2 \mathrm{~km}$} & $\mathrm{NC}$ & 11 & 4 \\
\hline & NL & 2438 & 19682 \\
\hline & IIC & 0.0048464 & 0.015474 \\
\hline & $\mathrm{PC}$ & 0.0074303 & 0.0475362 \\
\hline
\end{tabular}

Índices de conectividad: NC- número de componentes, NL- número de enlaces, IIC- índice de conectividad integral, PCíndice de probabilidad de conectividad.

Connectivity indices: NC- number of components, NL- number of links, IIC- integral index of connectivity, PC- probability of connectivity index.

De acuerdo con los índices de conectividad a nivel individual, y las categorías de importancia, hubo 367 unidades de muy alta importancia para la conectividad, cubriendo un área de 37 337.335ha (Fig. 2). El 80\% de estas fueron de selva manejada y $20 \%$ de vegetación natural conservada, principalmente selva mediana perennifolia y bosque mesófilo de montaña.

El fragmento con el mayor valor, para todas las distancias de desplazamiento y ambos índices de conectividad, fue el que representa el bloque más extenso de selva mediana perennifolia de la SLT, ubicado en la parte alta del volcán Santa Marta, entre los 650 y $1000 \mathrm{~m}$ de altura, con una superficie de 8489.329 ha.

De acuerdo a los tres componentes de los índices, ninguna de las unidades de muy alta importancia para la conectividad lo fue por el componente intra, es decir, que ninguno de los fragmentos de mayor área estaba aislado. Para rangos de dispersión largos (12km), las unidades adquirieron relevancia por el flujo potencial que representaron, en otras palabras, por el área y las relaciones topológicas que tenían. En ambos índices se explicó al menos el $78 \%$ de la importancia por este componente. Aunque un considerable número de elementos explicaron su papel por el componente de flujo, fue posible observar que algunos tenían cierta relevancia por los otros dos. El componente intra llegó a aportar un máximo de entre 10 y $20 \%$ del valor de los índices, y entre 2 y $5 \%$ del componente conector.

Para rangos de dispersión medio $(6 \mathrm{~km})$, el índice IIC indicó que todas las unidades de muy alta importancia lo fueron por su flujo potencial. El máximo aporte en el componente intra fue del $22 \%$ y para el componente conector el 3\%. De igual modo, el índice mostró que la mayoría de los casos debió su grado de relevancia al componente de flujo. Por otro lado, el índice PC arrojó que hubo 43 elementos que aportaron más a la conectividad del paisaje por ser puntos de conexión y el resto se definió por su flujo potencial. El $22 \%$ fue el aporte máximo del componente intra a la importancia de un fragmento. 
Para rangos de dispersión cortos $(2 \mathrm{~km})$ el índice IIC mostró tres fragmentos de alta importancia por ser conectores, el resto lo fue por su flujo potencial. El máximo aporte del componente intra fue de $26 \%$. Para el índice PC, 67 elementos fueron relevantes por su función conectora, el resto por su flujo potencial. El máximo aporte del componente intra fue de $9 \%$.

De las 70 unidades de hábitat viable que fueron importantes en la conectividad del paisaje por su papel como enlaces (Fig. 2), un $75 \%$ fueron de selva manejada, $20 \%$ de selva perennifolia (alta o mediana), $2.8 \%$ de manglar y $1.4 \%$ de bosque (mesófilo de montaña).

\section{DISCUSIÓN}

El alto número de fragmentos de vegetación natural de reducidas superficies, reflejan el proceso de deforestación que ha sufrido la SLT en las últimas décadas, proceso que se expresa como un mosaico dominante de potreros con manchones dispersos de selvas, manglares y bosques. Es importante notar que a pesar de esta problemática, la CONABIO reconoce la zona como de alto valor para la conservación, pues mantiene gran número de tipos de vegetación asociada a su amplio gradiente altitudinal (Arriaga et al., 2000). Castillo-Campos y Laborde (2004) identifican 22 tipos de vegetación y uso de suelo. Lo anterior se manifiesta en una gran diversidad de especies, en flora se reportan 3356 especies y 212 familias, de las cuales 1873 especies son de selva perennifolia, 1518 de bosques y 98 de manglares (Sosa \& Gómez-Pompa, 1994; Castillo-Campos \& Laborde, 2004); en lo referente a fauna, se señala la presencia de 139 especies de mamíferos, 565 de aves, 109 de reptiles, 49 de anfibios, 109 de peces y 1117 de insectos (Arriaga et al., 2000; CONANP, 2006).

La mayor parte de la superficie de vegetación natural está concentrada en relictos restringidos en la cima de los volcanes, si la presión de los sistemas productivos ubicados en terrenos bajos y laderas suaves no se reduce y se transforma en una matriz menos hostil, las áreas conservadas tenderán a segmentarse y aislarse. Es por tanto indispensable que se mantengan y fomenten las unidades de selva manejada, productivas para el hombre, que se asemejan en buena medida en estructura y composición a la vegetación natural. Los ecólogos Vandemeer, Perfecto, Philpott, y Chappell (2006) han hecho amplias propuestas sobre lo importante que es considerar el contexto paisajístico en el que se encuentran los relictos de vegetación conservada; su enfoque principal es que la matriz del paisaje debe servir como un puente entre la conservación de la biodiversidad con el desarrollo y bienestar de la población local.

Nuestro análisis demostró que la conectividad entre los fragmentos de vegetación natural conservada se incrementa cuando se toma en cuenta la selva manejada. Se debe notar que su importancia no solo radica en el hecho de mantener el paisaje funcionando como uno solo, sino en la función de proveer más enlaces, a modo de que existan más rutas de posible dispersión. Así, si un fragmento llegara a desaparecer el sistema no colapsa, pues tiene otras alternativas de flujo. Las especies de rango de desplazamiento corto, como mamíferos pequeños no voladores, por ejemplo la martucha (Potos flavus), tlacuache dorado (Caluromys derbianus) y la ardilla (Sciurus deppei deppei), se ven beneficiados, ya que, el paisaje está funcionalmente fragmentado cuando solo se considera la vegetación natural.

La conectividad en un paisaje depende en gran medida de la disponibilidad y la distribución del hábitat viable, en el caso que analizamos en este artículo aún es posible encontrar ambas condiciones. En la mayoría de las unidades de muy alta importancia lo son debido al flujo potencial que representan, es decir, a las dimensiones de área que ofrecen y al número de los posibles enlaces con otras áreas. Para las especies de rango de desplazamiento largo, como los felinos yaguaroundi (Herpailurus jaguarondi), tigrillo (Leopardus wiedii oaxacensis), ocelote (Leopardus pardalis pardalis), esto es fundamental para poder mantener sus poblaciones viables. 
Dado que es imposible frenar por completo la actividad productiva de los habitantes, se deben de impulsar actividades de menor impacto que mejoren la dinámica del paisaje, como lo son los sistemas tradicionales de manejo y los sistemas agroforestales. En la parte sur de la SLT, la población es predominantemente indígena, popoluca y nahua, asentada en un gran número de localidades dispersas, con pocos habitantes en cada una; en esta región la vegetación natural está mejor conservada si la comparamos con la parte norte, donde predomina la población mestiza (Guevara et al., 2000a). El presente análisis muestra que la mayor parte de los fragmentos de muy alta importancia para la conectividad son de selva manejada, reflejando la importancia de las prácticas productivas de bajo impacto para la continuidad de procesos ecológicos de la SLT. Aunque estas unidades son poco extensas en superficie, cumplen una función estratégica de amortiguamiento para los núcleos de las masas forestales conservadas y de enlace entre todos los elementos de paisaje. Esto favorece a especies animales, sobre todo si sus poblaciones son sensibles a las perturbaciones debido a que el efecto de borde se reduce. Las especies que se benefician de lo anterior son principalmente aquellas especies de rango de dispersión corto como el conejo de monte (Sylvilagus brasiliensis truei), ratón de campo (Heteromys desmarestianus lepturus), martucha (Potos flavus), entre otros. Algunas de las especies de dispersión media también se ven beneficiadas, como son principalmente el venado real (Odocoileus virginianus thomasi), mono araña (Ateles geoffroyi), coyote (Canis latrans cagottis), mono aullador (Alouatta palliatta). Estos espacios, en su mayoría de alta diversidad por la mezcla de especies de sistemas naturales y manejados, actúan como bancos de semillas y albergue de especies dispersoras y polinizadoras, que favorecen las dinámicas de ambos sistemas (Gliessman, 2002; Rands \& Whitney, 2011). En la SLT, el uso de estos espacios por ciertos grupos faunísticos (aves, murciélagos y primates) ha sido documentado en los trabajos de Estrada y Coates-Estrada (1995, 1996, 1997, 2001, 2005). En cuanto a la función que realizan como enlaces, hay que destacar que son claves los fragmentos que hacen posible mantener una ruta de intercambio entre las masas forestales principales, la cima del volcán San Martín, el volcán Santa Marta y el volcán Pajapan. Estas unidades de paisaje son relevantes y deben ser consideradas por cualquier proyecto de conservación en la zona, como potenciales corredores biológicos.

La zona Popoluca se caracteriza por tener poca ganadería extensiva y una agricultura moderada de autoconsumo, con el cultivo tradicional de milpa (cultivo mixto de maíz, frijol y calabaza principalmente), en un sistema de roza- tumba- quema. El café es un producto comercial, sembrado bajo dosel de selva, alternándose con los acahuales (Paré et al., 1994). Estas prácticas selectivas de elementos de la selva, que dejan árboles dispersos ya sea dosel o cercas vivas, pudieran favorecer la resiliencia de la selva; en la medida que las alteraciones disminuyan y su potencial ecológico se exprese totalmente. Existen trabajos que documentan como los árboles de selva que se dejan dispersos en potreros y zonas agrícolas (Guevara, Meave, Moreno-Casassola, Laborde \& Castillo, 1994; Guevara et al., 2000b; Harvey et al., 2006), las cercas vivas (Harvey et al., 2005; Chacón \& Harvey, 2006) y los agrosistemas (Estrada, et al., 2005), favorecen la conservación de diversidad animal (al proveer hábitat y recursos), la dispersión de especies y la conectividad estructural de un paisaje fragmentado.

En conclusión podemos decir, que para que la conservación en la SLT sea viable a largo plazo, los esfuerzos para su conservación deben de llevarse hasta la escala del paisaje, ir más allá de los límites del área protegida e incluir sistemas tradicionales del manejo de la selva. Los sistemas tradicionales de manejo son una opción viable para fortalecer las prácticas productivas en el sentido de reducir el impacto que se ejerce sobre las dinámicas del paisaje. Otras prácticas como son los sistemas agroforestales, silvopastoriles, huertos tradicionales, cercas vivas, etc. cumplen el mismo papel, y por tanto, también deben promoverse para consolidar el funcionamiento del paisaje. El apoyo 
brindado a los sistemas tradicionales revaloriza el conocimiento y cultura indígenas y campesinos, que son parte de la riqueza nacional. Estudios de ecología de paisaje mediante el análisis por medio de SIG's y la conectividad de los hábitats viables es imprescindible. Con base en ellos se puede estudiar la complejidad a un nivel de escala pequeña y la dinámica de la fragmentación de unidades de vegetación. En nuestra investigación se pudo establecer las áreas claves que conforman redes potenciales para la conservación. El modelo espacial resultante de nuestro análisis establece la importancia del manejo de los campesinos popolucas, como elemento básico para entender las características actuales de los bosques y selvas de la SLT abriendo nuevas estrategias de análisis para su conservación y sustentabilidad.

\section{AGRADECIMIENTOS}

Agradecemos al Dr. Mario M. Aliphat Fernández, director del proyecto "Huertos y cacaotales", SEP-CONACYT $131026 \mathrm{H}$, el apoyo académico y financiero para la realización del presente trabajo.

\section{RESUMEN}

La existencia cada vez más frecuente de mosaicos de paisaje se expresa como una matriz circundante de actividades agropecuarias que enmarcan relictos de vegetación primaria. Cualquier acción de conservación debe de estar orientada a establecer o incrementar aquellos sistemas interactivos que mantengan los flujos del paisaje a través de enlaces. Los espacios ocupados por sistemas tradicionales de manejo favorecen y mantienen esta función. En el presente trabajo se evaluó, a través de un sistema de información geográfica, la importancia de las unidades de selva manejada de manera tradicional (acahuales-cafetales) en la conectividad del paisaje en la zona indígena Popoluca de la Sierra de los Tuxtlas, México. El material cartográfico utilizado para determinar los tipos de vegetación y su cobertura, abarca el periodo 1991-2008. Se emplearon cuatro índices para evaluar la conectividad a nivel paisaje y detectar cuáles son los fragmentos de muy alta prioridad para su mantenimiento. A nivel individual los índices evaluaron si los fragmentos son importantes por su área, por su flujo potencial o por su función conectora. Los resultados muestran que el paisaje funciona como un solo sistema, con baja conectividad. Los valores mejoran al incluirse la selva manejada como hábitat viable. Se detectaron 367 fragmentos de muy alta prioridad, $80 \%$ de ellos de selva manejada. Los fragmentos en su mayoría fueron importantes por el flujo potencial que representan (dimensiones y relaciones topológicas). Solo 70 fragmentos fueron importantes por su función como conectores, éstos actúan como corredores con las masas forestales de mayor tamaño localizadas en la cima de los volcanes, y son principalmente fragmentos de selva manejada (75\%). Se concluye que las unidades de selva manejada de manera tradicional juegan un papel significante en el mantenimiento de la conectividad del paisaje.

Palabras clave: selva, acahuales, conectividad del paisaje, manejo tradicional Popoluca, Los Tuxtlas.

\section{REFERENCIAS}

Altieri, M. A. (1991). ¿Por qué estudiar la agricultura tradicional? Agroecología y Desarrollo, 1(1), 16-24.

Altieri, M. A. (2004). Linking ecologist and traditional farmers in the search for sustainable agriculture. Frontiers in ecology and the environment, 2(1), 35-42.

Anderson, E. (2003). Traditional knowledge of plant resources. In A. Gómez Pompa, F. Allen, S. L. Fedick, \& J. J. Jiménez-Osornio (Eds.), The lowland Maya area: three millennia at the human-wildland interface (pp. 533-550). Nueva York: The Haworth Press.

Arriaga Cabrera, L., Espinoza-Rodríguez, J. M., AguilarZúñiga, C., Martínez-Romero, E., Gómez-Mendoza, L., \& Loa Loza, E. (Coords.). (2000). Regiones terrestres prioritarias de México. México: CONABIO.

Arroyo-Rodríguez, V. \& Mandujano, S. (2006). The importance of tropical rain forest fragments to the conservation of plant species diversity in Los Tuxtlas, Mexico. Biodiversity and conservation, 15, 4159-4179.

Bennett, A. F. (2004). Enlazando el paisaje: El papel de los corredores y la conectividad en la conservación de la vida silvestre. San José: UICN.

Berkes, F., Colding, J., \& Folke, C. (2000). Rediscovery of traditional ecological knowledge as adaptative management. Ecological Applications, 10(5), 1251-1262.

Boege Schmidt, E. (2008). El patrimonio biocultural de los pueblos indígenas de México. México: INAH-CDI.

Castillo-Campos, G. \& Laborde, J. (2004). La vegetación. In S. Guevara Sada, J. Laborde Dovalí, \& G. Sánchez-Ríos (Eds.), Los Tuxtlas. El paisaje de la sierra (pp. 231-270). México: INECOL-Unión Europea.

CDI. (2006). Regiones indígenas de México. México: CDI-PNUD.

Chacón, M. \& Harvey, C. (2006). Live fences and landscape connectivity in a Neotropical agricultural landscape. Agroforestry Systems, 68, 15-26. 
Chazdon, R. L., Harvey, C. A., Komar, O., Griffith, D. M., Ferguson, B. G., Martínez-Ramos, M., Morales, H., Nigh, R., Soto-Pinto, L., van Breugel, M., \& Philpott, S. M. (2009). Beyond reserves: A research agenda for conserving biodiversity in human- modified tropical landscapes. Biotropica, 41(2), 142-153.

CONANP. (2006). Programa de conservación y manejo Reserva de la Biósfera Los Tuxtlas. México: CONANP-SEMARNAT.

Estrada, A. \& Coates-Estrada, R. (1995). Las selvas tropicales húmedas de México. Recurso poderoso, pero vulnerable. México: Fondo de Cultura Económica.

Estrada, A. \& Coates-Estrada, R. (1996). Tropical Rain Forest Fragmentation and wild populations of primates at Los Tuxtlas, Mexico. International journal of primatology, 17(5), 759-783.

Estrada, A. \& Coates-Estrada, R. (1997). Anthropogenic landscape changes and avian diversity at Los Tuxtlas, Mexico. Biodiversity and conservation, 6, 19-43.

Estrada, A. \& Coates-Estrada, R. (2001). Bat species richness in live fences and in corridors of remnants forest vegetation at Los Tuxtlas, Mexico. Ecography, 24, 94-102.

Estrada, A. \& Coates-Estrada, R. (2005). Diversity of neotropical migratory landbird species assemblages in forest fragments and man-made vegetation in Los Tuxtlas, Mexico. Biodiversity and conservation, 14, 1719-1734.

Estrada, A., Harvey, C., Sáenz, J., Muñoz, D., Naranjo, E., \& Rosales-Meda, M. (2005). Valor de algunas prácticas para la conservación de poblaciones de primates en paisajes fragmentados en Mesoamérica. Universidad y ciencia, No. especial (II), 85-94.

Gadgil, M., Berkes, F., \& Folke, C. (1993). Indigenous knowledge for biodiversity conservation. Ambio, 22(2-3), 151-156.

Gliessman, S. R. (2002). Agroecología: procesos ecológicos en agricultura sostenible. Turrialba: CATIE.

Gliessman, S. R., Rosado-May, F., Guadarrama-Zugasti, C., Jedlicka, J., Cohn, A., Méndez, E., Cohen, R., Trujillo, L., Bacon, C., \& Jaffe, R. (2007). Agroecología: promoviendo una transición hacia la sostenibilidad. Ecosistemas, 16(1), 13-23.

Gómez Pompa, A., \& Kaus, A. (1998). From prehispanic to future conservation alternatives: Lessons from Mexico. Proceedings of the National Academy of Sciences, 96, 5982-5986.

González, E., Dirzo, R., \& Vogt, R. (Eds.). (1997). Historia Natural de Los Tuxtlas. México: UNAM.

González, A. \& Vicario, H. (1998). Mamíferos veracruzanos en vías de extinción. Veracruz: IVEC.

Guevara Sada, S., Meave, J., Moreno-Casassola, P., Laborde, J., \& Castillo, S. (1994). Vegetación y flora de potreros en la Sierra de Los Tuxtlas, México. Acta Botánica Mexicana, 28, 1-27.

Guevara Sada, S., Laborde Dovalí, J., \& Sánchez Ríos, G. (2000a). La reserva de la biósfera Los Tuxtlas, México. (Documento de trabajo núm. 29). Paris: UNESCO.

Guevara Sada, S., Laborde Dovalí, J., \& Sánchez Ríos, G. (2000b). Are isolated remnant trees in pastures a fragmented canopy?. Selbyana, 19(1), 34-43.

Guevara Sada, S., Sánchez Ríos, G., \& Landgrave, R. (2004). La deforestación. In S. Guevara Sada, J. Laborde Dovalí, \& G. Sánchez-Ríos (Eds.), Los Tuxtlas. El paisaje de la sierra (pp. 85-110). México: INECOL-Unión Europea.

Harvey, C., Villanueva, C., Villacís, J., Chacón, M., Muñoz, D., López, M., Ibrahim, M., Gómez, R., Taylor, R., Martínez, J., Navas, A., Saenz, J., Sánchez, D., Medina, A., Vílchez, S., Hernández, B., Pérez, A., Ruiz, F., López, F., Lang, I., \& Sinclair, F. (2005). Contribution of live fences to the ecological integrity of agricultural landscapes. Agriculture, ecosystems and environment, 111, 200-230.

Harvey, C., Medina, A., Merlo, D., Vílchez, S., Hernández, B., Saenz, J., Maes, J., Casanoves, F., \& Sinclair, F. (2006). Patterns of animal diversity in different forms of tree cover in agricultural landscapes. Ecological applications, 16(5), 1986-1999.

INEGI. (2008). Ortofotografia digital de la zona de Los Tuxtlas. Escala 1:40 000. México: INEGI.

Kettunen, M., Terry, A., Tucker, G., \& Jones A. (2007). Guidance on the maintenance of landscape connectivity features of major importance for wild flora and fauna- Guidance on the implementation of article 3 of the Birds Directive (79/409/EEC) and article 10 of the Habitats Directive (92/43/EEC). Bruselas: IEEP.

Lewis, M. P., Simons, G. F., \& Fenning, C. D. (Eds.). (2013). Ethnologue: Languages of the World. Dallas: SIL International. Recuperado de http://www.ethnologue.com/

McGarigal, K., (2014). Fragstats v4: Spatial Pattern Analysis Program for Categorical and Continuous Maps-Help manual. Amherst: University of Massachusetts. Recuperado de http://www.umass.edu/ landeco/research/fragstats/fragstats.html

McIntyre, S. \& Hobbs, R. (1999). A framework for conceptualizing human effects on landscapes and its relevance to management and research models. Conservation Biology, 13(6), 1282-1292.

Neel, M. (2008). Patch connectivity and genetic diversity conservation in the federally endangered and narrowly endemic plant species Astragalus albens (Fabaceae). Biological Conservation, 141, 938-955.

Paré, L. (1994). Alternate sustainable development strategy for a biosphere reserve, Sierra de Santa Marta, 
Veracruz, Mexico. In T. Roach (Ed.), Sustainable mountain agriculture. An international workshop held at the international centre for research in agroforestry, Nairobi, Kenya (pp. 25-34). Nairobi: IDRC.

Paré, L., Agüero, J. C., \& Blanco, J. L. (1994). Diagnóstico de la producción del maíz en la Sierra de Santa Marta. In D. J. Buckles, C. P. Arcos, G. Díaz, J. Cruz, \& H. Narave (Eds.), Memorias del taller sobre las politicas para una agricultura sustentable en la Sierra de los Tuxtlas y Santa Marta, Veracruz (pp. 17-35). México: CIMMYT.

Pascual-Hortal, L. \& Saura, S. (2006). Comparison and development of new graph-based landscape connectivity indices: towards the priorization of habitat patches and corridors for conservation. Landscape Ecology, 21(7), 959-967.

Pascual-Hortal, L. \& Saura, S. (2007). Impact of spatial scale on the identification of critical habitat patches for the maintenance of landscape connectivity. Landscape and Urban Planning, 83(2-3), 176-186.

Pohle, P. \& Gerique, A. (2006). Traditional ecological knowledge and biodiversity management in the Andes of Southern Ecuador. Geographica Helvetica, 4, 275-285.

Proyecto Sierra de Santa Marta, A.C.-PSSM (2011). Actualización de la tasa de cambio del uso del suelo en la reserva de la biósfera Los Tuxtlas. México: CONANP.

Rands, S. A. \& Whitney, H. M. (2011). Field margins, foraging distances and their impacts on nesting pollinator success. PLoS ONE 6(10), e25971.

Reyes-García, V., Martí, N., McDade, T., Tanner, S., \& Vadez, V. (2007). Concepts and methods in studies measuring individual ethnobotanical knowledge. Journal of ethnobiology, 27(2), 182-203.

Santos, T. \& Tellería, J. L. (2006). Pérdida y fragmentación del hábitat: efecto sobre la conservación de las especies. Ecosistemas, 15(2), 3-12.

Saura, S. \& Pascual-Hortal, L. (2007). A new habitat availability index to integrate connectivity in landscape conservation planning: comparison with existing indices and application to a case study. Landscape and Urban Planning, 83(2-3), 91-103.

Saura, S. \& Torné, J. (2009). Conefor Sensinode 2.2: a software package for quantifying the importance of habitat patches for landscape connectivity. Environmental Modelling \& Software, 24, 135-139.

Saura, S. \& Rubio, L. (2010). A common currency for the different ways in which patches and links can contribute to habitat availability and connectivity in the landscape. Ecography, 33, 523-537.

Secretaría del Medio Ambiente y Recursos Naturales SEMARNAT. (1998). Decreto por el que se declara área natural protegida, con carácter de reserva de la biosfera, la región denominada Los Tuxtlas. México: Diario Oficial de la Federación.

Sosa, V. \& Gómez-Pompa, A. (Comps.). (1994). Lista florística. Flora de Veracruz-Fascículo 82. Veracruz: Instituto de Ecología, A.C.

Toledo, V. M., Ortiz, B., \& Medellín-Morales, S. (1994). Biodiversity islands in a sea of pastureland: indigenous resource management in the humid tropics of Mexico. Etnoecológica, 3, 37-50.

Toledo, V. M., Alarcón-Chaires, P., Moguel, P., Olivo, M., Cabrera, A., Leyequien, E., \& Rodríguez-Aldabe, A. (2001). El atlas etnoecológico de México y Centroamérica: fundamentos, métodos y resultados. Etnoecológica, 6(8), 7-41.

Toledo, V. M., Ortiz-Espejel, B., Cortés, L., Moguel, P., \& Ordoñez, M. J. (2003). The multiple use of tropical forest by indigenous peoples in Mexico: a case of adaptative management. Conservation Ecology, $7(3), 9$.

Vandemeer, J., Perfecto, I., Philpott, S., \& Chappell, M. (2006). Reenfocando la conservación en el paisaje: La importancia de la matriz. In J. Sáenz \& C. Harvey (Eds.), Evaluación y conservación de la biodiversidad en paisajes fragmentados en Mesoamérica (pp. 75-104). San José: Universidad Autónoma de Costa Rica.

Velázquez, E., (2001). El territorio de los popolucas de Soteapan, Veracruz: Transformaciones en la organización y apropiación del espacio. Relaciones, 22(87), $15-48$.

Villavicencio García, R., Saura Martínez de Toda, S., Santiago Pérez, A. L., \& Chávez Hernández, A. (2009). La conectividad forestal de las áreas protegidas del estado de Jalisco con otros ambientes naturales. Scientia-CUCBA, 11(1-2), 43-50. 
